\title{
Analysis of DevOps Tools to Predict an Optimized Pipeline by Adding Weightage for Parameters
}

\author{
R. Vaasanthi \\ Research Scholar, \\ SCSVMV University \\ Kanchipuram
}

\author{
V. Prasanna Kumari, PhD \\ HOD, MCA \\ Rajalakshmi Engineering \\ College, Chennai
}

\author{
S. Philip Kingston \\ Project Manager \\ Infosys, Mahindra City, \\ Chennai
}

\begin{abstract}
Now-a-days more than ever, DevOps [Development + Operations] has gained a tremendous amount of attention in software industry. Selecting the tools for building the DevOps pipeline is not a trivial exercise as there are plethora's of tools available in market. It requires thought, planning, and preferably enough time to investigate and consult other people. Unfortunately, there isn't enough time in the day to dig for top-rated DevOps tools and its compatibility with other tools. Each tool has its own pros/cons and compatibility of integrating with other tools. The objective of this paper is to propose an approach by adding weightage to each parameter for the curated list of the DevOps tools.
\end{abstract}

\section{Keywords}

DevOps, SCM, dependencies, compatibility and pipeline

\section{INTRODUCTION}

DevOps, where automation and orchestration work hand in hand to streamline deployment contains numerous tools, so researching which one is right for your application is vital. The available DevOps tools can be broadly classified [1] by using Data Mining techniques or by periodic table. The periodic table, or periodic table of elements, is a tabular arrangement of the chemical elements, ordered by their atomic number, electron configuration, and recurring chemical properties, whose structure shows periodic trends [2]. Similarly, DevOps tools are arranged by different software companies. For instance, Xebialabs - An independent software company specializing in DevOps and CyberArk a publicly traded information security company [3]. Each project has a continuing pressure to adopt DevOps to increase the productivity, time to market, etc.

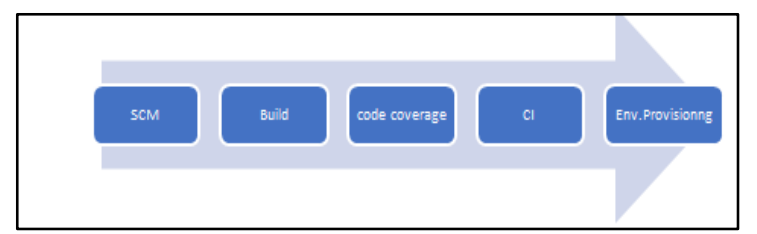

Fig1. Basic Pipeline

DevOps pipeline is classified with SCM [Source Code Management], Build, CI [Continuous Integration], Code Coverage and Environment provisioning tools to build a pipeline. Each classification has its own categories/parameters. The study described here was undertaken to contribute to the small set of DevOps tools. For each classification of tools, weightage is added to the listed parameters - License, Operating system, Platform, Hardware and configuration, Tool/Framework, Dependencies, Embedded GUI, Performance, Migration Feasibility, scalability, Compatibility to CICD pipelines, Security, Reliability, Support availability and its availability over the cloud.

\section{SCM}

Source code management (SCM) is a software tool used for development, versioning and enables team working in multiple locations to work together more effectively. This plays a vital role in increasing team's productivity. Some of the SCM tools, considered for this study are GIT, SVN, CVS, ClearCase, Mercurial, TFS, Monotone, Bitkeeper, Code coop, Darcs, Endevor, Fossil, Perforce, Rational Synergy, Source Safe, and GNU Bazaar. Table1 consists of SCM tools with weightage.

\section{BUILD}

Build is a process that enables source code to be automatically compiled into binaries including code level unit testing to ensure individual pieces of code behave as expected [4]. The most famous build tools considered here are Maven, Ant, Gradle, MS build, Ubuild, phing and Nant. Table 2 consists of Build tools with weightage.

\section{CI}

Continuous Integration is compiling, building, packaging and integrating the software on a continuous basis. With every check-in of a source code, system triggers the compilation process and runs code coverage, Unit testing, static analysis and automated deployment. Some of the CI tools considered here are Jenkins, Buildbot, Travis, Strider CI, Bamboo, Teamcity, Solano CI, CruiseControl and Semaphore. Table 3 consists of CI tools with weightage.

\section{CODE COVERAGE}

Code coverage testing is to determine which blocks of source code are executed during run time in an operating system is accomplished using instrumented code and a performance analysis profiler [5]. This can also be used to cover all code snippets in the program for testing. Some of the code coverage tools are EMMA, Hansel, Cobertura, Clover, GroboUtils, Quilt, Jcoverage, Clover.NET, Ncover, DevPartner, C\# Test Coverage, Squish Coco, Dot Cover, Pylid, PHP Test Coverage Tool. Table 4 consists of code coverage tool with weightage.

\section{ENIVRONMENT PROVISIONING}

Environment provisioning is a key part of a continuous delivery process. The idea is simple: we should not only build, test and deploy application code, but also the underlying application environment. Environment consists of three main areas Infrastructure, configuration and dependencies [6]. Some of the tools used are Ansible, Cobbler, Chef, Puppet, Salt Stack, Windows PowerShell, CFEngine, Rex, Cloudify and Pallet.Table 5 consits of tools with weightage. 


\section{HOW TO SELECT TOOL?}

Face to Face, Telephone discussions and e-mail questionnaires method are used to understand client requirements. The design, wording, form and order of questions can affect the type of tool obtained while selecting the pipeline manually. In addition, by adding the weightages after selecting all the tools can be used to build an optimized pipeline. Unless all the pieces of software delivery lifecycle work like a well-oiled machine-efficiency of organization to optimize the delivery, lifecycle cannot be met [7]. The problem is to identify the optimized tool set considering the future directions and growth for the company for the above cluster of tools. As coined by someone "To make error is human. To propagate error to all server in automatic way is DevOps". To identify this manually needs lot of knowledge/patience/time on all the tools and still possibilities are there to miss out the optimized set.

\section{CONCLUSION}

To recapitulate, optimized tool set can be identified with the least weightage calculated based on the templates for each SCM, build, CI, code coverage and Environment provisioning. The future study of this paper is to automate the addition of the weightage process. The DevOps pipeline can be expanded to repository management, plan, Testing and Deployment

\section{REFERENCES}

[1] R. Vaasanthi, Prasanna V Kumari, S. Philip Kingston "Analysis of DevOps Tools using the Traditional Data Mining Techniques" International Journal of Computer Applications 161(11):47-49, March 2017.

[2] https://en.wikipedia.org/wiki/Periodic_table

[3] https://www.cyberark.com/blog/tag/periodic-table-ofdevops-tools/ June 27, 2018.

[4] R. Vaasanthi, Philip S Kingston and Prasanna V Kumari. Comparative Study of DevOps Build Automation Tools. International Journal of Computer Applications 170(7):58, July 2017.

[5] United states Patent Application Publication, Chen et al. Pub. No.: US 2009/0287729 A1. Pub. Date: Nov. 19, 2009.

[6] Rodrigo Gonzale, "https://clarive.com/why-environmentprovisioning/" March 19,2018

[7] Manish Virmani," Understanding DevOps\& bridging the gap from continuous integration to continuous delivery" Innovative computing Technology (INTECH),2015 Fifth International Conference on 20-22 May 2015.

\section{APPENDIX}

Table1: SCM tools with categories and weightag

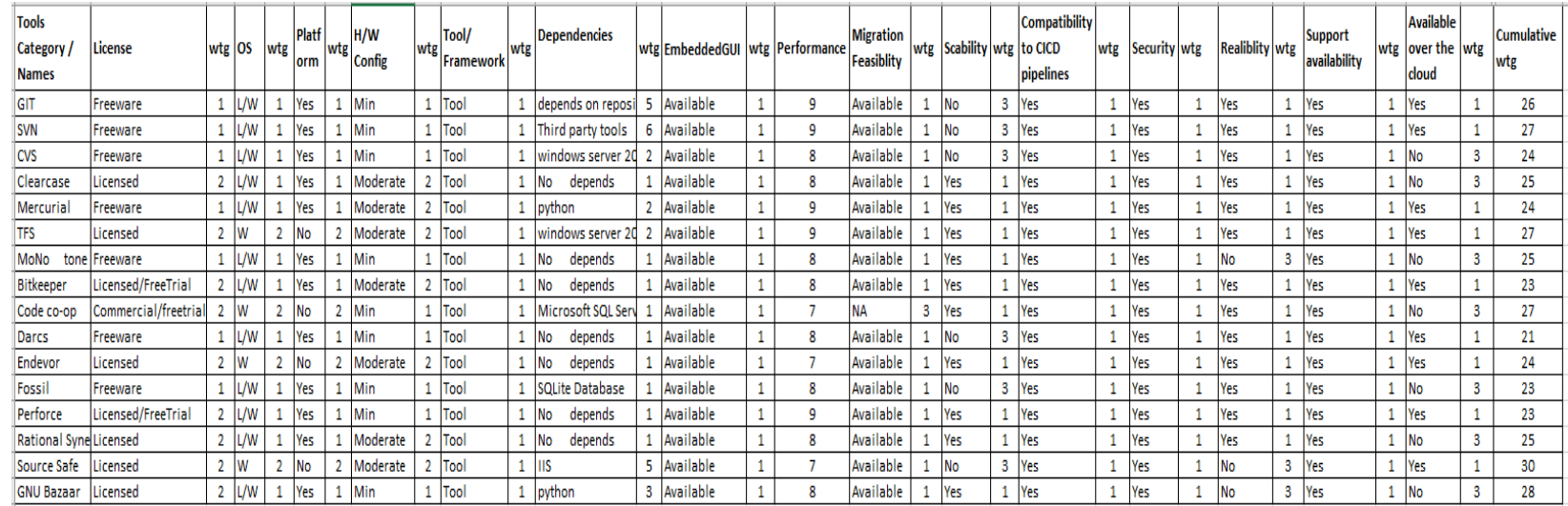

Table 2: Build tools with categories and weightage

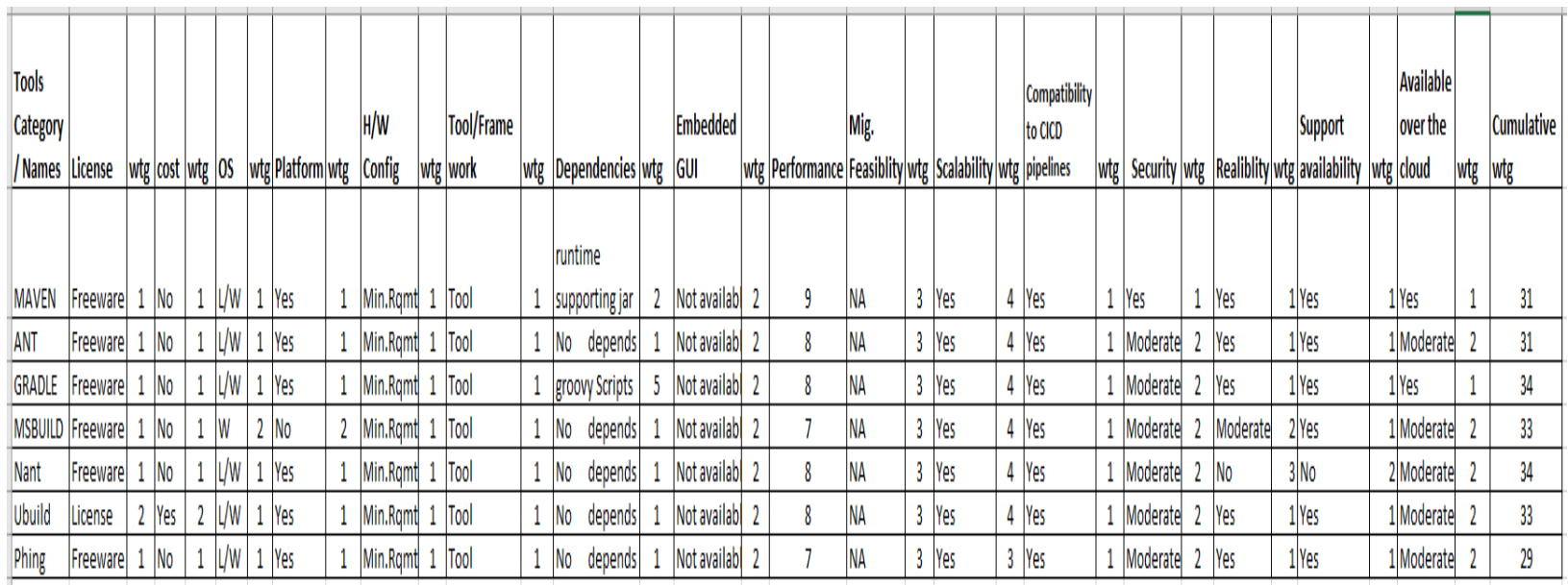


Table 3: CI tools with categories and weightage

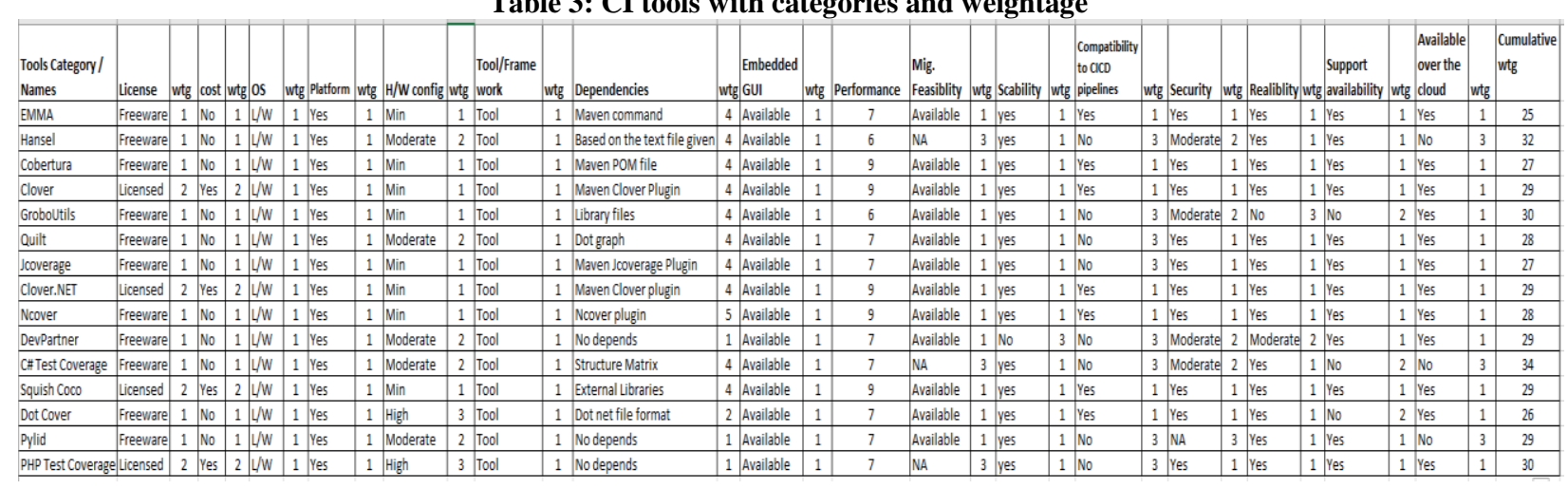

Table 4: Code Coverage tools with categories and weightage

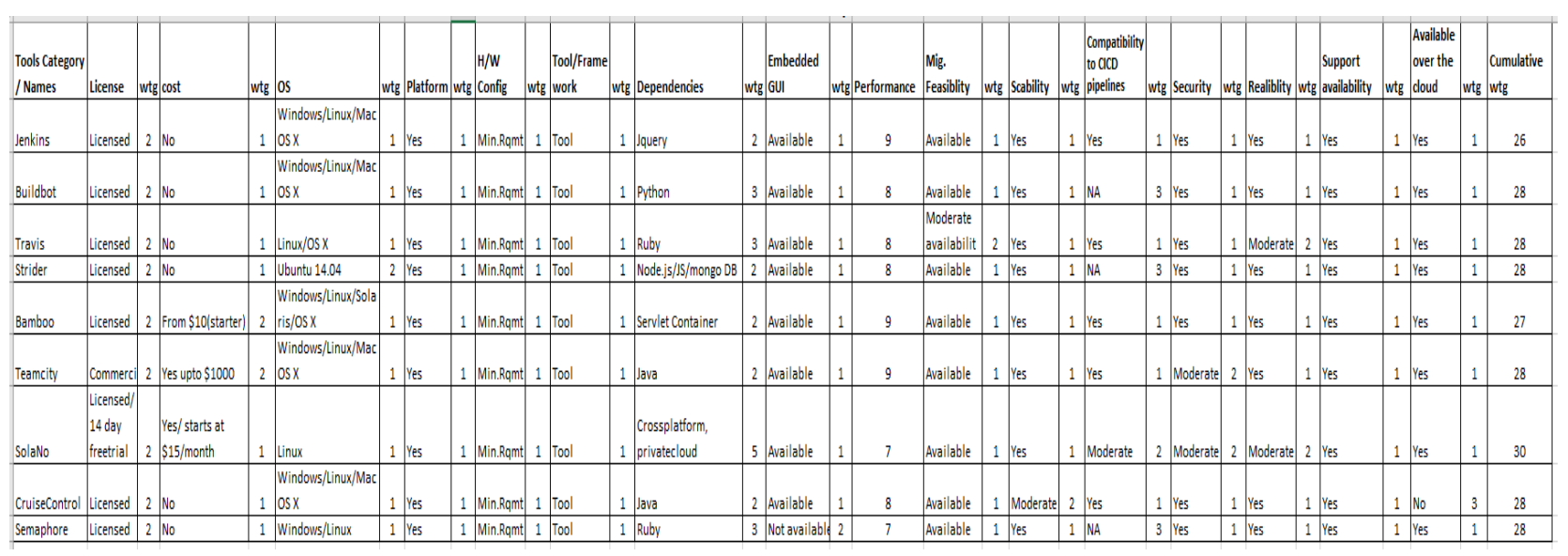

Table 5: Environment provisioning tools with categories and weightage

\begin{tabular}{|c|c|c|c|c|c|c|c|c|c|c|c|c|c|c|c|c|c|c|c|c|c|}
\hline $\begin{array}{l}\text { Tools Gategory } \\
\text { / Names }\end{array}$ & License & wtg & cost & wtg & & wtg |lattorm & \begin{tabular}{l|l}
$\mathrm{wt} / \mathrm{W}$ \\
wignfig
\end{tabular} & \begin{tabular}{|l|l|} 
witg wool/Frame \\
witk
\end{tabular} & wtg Dependencies & \begin{tabular}{|l|l|} 
wig Gubedded \\
G Gul
\end{tabular} & wtg $P$ & Performance & $\begin{array}{l}\text { Mig. } \\
\text { Feasiblity }\end{array}$ & wig scability & \begin{tabular}{|l|l} 
Compartility \\
to cico \\
wtg pipelines
\end{tabular} & wtg Seanity & wtg Realiblity w & \begin{tabular}{l|l} 
& Support \\
wtg avalability
\end{tabular} & wtg $\begin{array}{l}\text { Avallable } \\
\text { over the } \\
\text { cloud }\end{array}$ & & $\begin{array}{l}\text { Cumulative } \\
\text { wtg }\end{array}$ \\
\hline Jenkins & Licensed & 2 & & 1 & $\begin{array}{l}\text { Windows/Linux/Mac } \\
\text { os } x\end{array}$ & \begin{tabular}{|l|l|}
1 & \\
\end{tabular} & 1 Min.Rqm: & 1 Tool & 1 lquery & 2 Avvilable & 1 & 9 & Available & 1 Yes & 1 Yes & 1 Yes & 1 Yes & 1 Yes & 1 Yes & 1 & 26 \\
\hline Buildbot & Licensed & 2 & & 1 & \begin{tabular}{|l|} 
Window//Linux/Mac \\
os $x$
\end{tabular} & \begin{tabular}{|l|l|}
1 & Yes \\
\end{tabular} & 1 Min.Rant: & 1 Tool & 1 Python & \begin{tabular}{|l|l|}
3 & Available \\
\end{tabular} & 1 & 8 & Available & 1 Yes & 1 NA & $\begin{array}{l}3 \text { Yes } \\
\end{array}$ & 1 Yes & 1 Yes & 1 Yes & 1 & 28 \\
\hline Travis & Licensed & 2 & & 1 & Linux/DSX & 1 Yes & 1 Min.Ramt: & \begin{tabular}{|l|l|} 
& Tool \\
\end{tabular} & 1 Ruby & \begin{tabular}{l|l}
3 & Available \\
\end{tabular} & 1 & 8 & $\begin{array}{l}\text { Modererate } \\
\text { avaliabilit }\end{array}$ & 2 yes & 1 Yes & 1 Yes & 1 Moderate & 2 Yes & 1 Yes & 1 & 28 \\
\hline Strider & Licensed & 2 & No & 1 & Ubbuntu 1404 & \begin{tabular}{|l|l|}
2 & Yyes \\
\end{tabular} & \begin{tabular}{|l|l|}
1 & Min.Rant: \\
\end{tabular} & \begin{tabular}{|l|l|}
1 & Tool \\
\end{tabular} & \begin{tabular}{|l|l|l|}
1 & Node. $5 / 1 / 1 /$ mongo \\
\end{tabular} & \begin{tabular}{|l|l|}
2 & Available \\
\end{tabular} & 1 & 8 & \begin{tabular}{|l|l|} 
Available \\
\end{tabular} & \begin{tabular}{|l|l|}
1 & Yes \\
\end{tabular} & \begin{tabular}{l|l|}
1 & $N A$ \\
\end{tabular} & \begin{tabular}{|l|l|}
3 & Yes \\
\end{tabular} & \begin{tabular}{l|l|l|}
1 & Yes \\
\end{tabular} & \begin{tabular}{|l|l|}
1 & Yyes \\
\end{tabular} & \begin{tabular}{l|l|l}
1 & Yes \\
\end{tabular} & 1 & 28 \\
\hline Bamboo & Licensed & 2 & From $\$ 10 \mid$ starter) & 2 & \begin{tabular}{|l|}
$\begin{array}{l}\text { Windows/Linux/sola } \\
\text { ris/oSX }\end{array}$ \\
\end{tabular} & \begin{tabular}{|l|l|}
1 & Yes \\
\end{tabular} & 1. Min.Ram: & \begin{tabular}{|l|l|l|} 
& Tool \\
\end{tabular} & 1 Servet Container & \begin{tabular}{|l|l|}
2 & Available \\
\end{tabular} & 1 & 9 & Avaliable & 1 Yes & 1 Yes & 1 Yes & 1 Yes & 1 Yes & 1 Yes & 1 & 27 \\
\hline Teamcity & Commerc & 2 & Yes upto $\$ 1000$ & 2 & $\begin{array}{l}\text { Windows/Linux/Mac } \\
\text { osX }\end{array}$ & \begin{tabular}{|l|l|}
1 & Yes \\
\end{tabular} & 1 Min.Ramt & 1 Tool & 1 lava & 2 Available & 1 & 9 & Available & 1 Yes & 1 Yes & 1 Moderate & 2 Yes & 1 Yes & 1 Yes & 1 & 28 \\
\hline Solano & \begin{tabular}{|l} 
Licensed// \\
14 day \\
freetrial
\end{tabular} & 2 & $\begin{array}{l}\text { Yes/ starts at } \\
\$ 15 / \text { month }\end{array}$ & 1 & Linux & 1 Yes & 1 Min.Rqmit & 1 Tool & 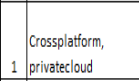 & \begin{tabular}{|l|l|l}
5 & Avilable \\
\end{tabular} & 1 & 7 & Available & 1 Yes & 1 Moderate & 2 Moderate & \begin{tabular}{|l|l|} 
& Moderate \\
\end{tabular} & $\begin{array}{l}2 \text { Yes } \\
\end{array}$ & 11 Yes & 1 & 30 \\
\hline Cruisecontrol L & Licensed & 2 & & 1 & $\begin{array}{l}\text { Windows/Linux/Mac } \\
\text { OSX }\end{array}$ & \begin{tabular}{|l|l}
1 & \\
Yes
\end{tabular} & 1 Min.Ramt: & \begin{tabular}{|l|l|} 
& Tool \\
\end{tabular} & 1 lava & 2 Available & 1. & 8 & Avaliable & 1 Moderate & \begin{tabular}{|l|l|l|}
2 & Yes \\
\end{tabular} & 1 Yes & 1 Yes & 1 Yes & 1 No & 3 & 28 \\
\hline Semaphore & Licensed & 2 & & 1 & \begin{tabular}{|l|} 
Windows/Linux \\
\end{tabular} & \begin{tabular}{|l|l|l|}
1 & Yyes \\
\end{tabular} & \begin{tabular}{l|l|}
1 & Min.Rqut: \\
\end{tabular} & \begin{tabular}{|l|l|}
1 & Tool \\
\end{tabular} & \begin{tabular}{|l|l|}
1 & Ruby \\
\end{tabular} & \begin{tabular}{|c|c|c|}
3 & Not availabll \\
\end{tabular} & 2 & 7 & Available & \begin{tabular}{|l|l|}
1 & Yes \\
\end{tabular} & \begin{tabular}{|l|l|}
1 & $\mathrm{NA}$ \\
\end{tabular} & \begin{tabular}{|l|l|}
3 & Yes \\
\end{tabular} & \begin{tabular}{l|l|l|}
1 & Yes \\
\end{tabular} & \begin{tabular}{|l|l|}
1 & Yes \\
\end{tabular} & \begin{tabular}{l|l}
1 & Yes \\
\end{tabular} & 1 & 28 \\
\hline
\end{tabular}

